\title{
Application of vancomycin-impregnated calcium sulfate hemihydrate/nano- hydroxyapatite/chitosan injectable hydrogels combined with BMSCs sheet for the treatment of infected bone defects in rabbit model
}

\section{Yanjun Wang}

Department of Orthopaedics, Second affiliated hospital, Air Force Medical University

Zihou Zhao

Department of Orthopaedics, Second affiliated hospital, Air Force Medical University

Shiyu Liu

Institute of Oral Tissue Engineering, Air Force Medical University

Wen Luo

Department of Ultrasound, Xijing Hospital, Air Force Medical University

Guoliang Wang

Department of Orthopaedics, Second affiliated hospital, Air Force Medical University Zhenfeng Zhu

Department of Orthopaedics, Second affiliated hospital, Air Force Medical University Qiong Ma

Department of Orthopaedics, Second affiliated hospital, Air Force Medical University Yunyan Liu

Department of Orthopaedics, Second affiliated hospital, Air Force Medical University Linhu Wang

Department of Orthopaedics, Second affiliated hospital, Air Force Medical University

Shuaikun Lu

Department of Orthopaedics, Second affiliated hospital, Air Force Medical University

\section{Yong Zhang}

Department of Orthopaedics, Second affiliated hospital, Air Force Medical University Jixian Qian

Department of Orthopaedics, Second affiliated hospital, Air Force Medical University

Yunfei Zhang ( $\nabla$ tdbone@163.com )

Department of Orthopaedics, Second affiliated hospital, Air Force Medical University 
Keywords: Nano-hydroxyapatite, Calcium sulfate hemihydrate, Chitosan, Bone marrow mesenchyml stem cell, Osteomyelitis

Posted Date: February 24th, 2022

DOI: https://doi.org/10.21203/rs.3.rs-1364846/v1

License: (1) This work is licensed under a Creative Commons Attribution 4.0 International License. Read Full License 


\section{Abstract \\ Background}

Choice of bone substitutes for the therapy of infected bone defects (IBDs) attracts surgeons' attention for years. However, single-stage bioabsorbable material that provides carriers for antibiotic releasing as well as scaffold for BMSCs sheet need further exploration. Our study was designed to investigate the effect of vancomycin-loaded calcium sulfate hemihydrate/nano-hydroxyapatite/chitosan (CSH/n-HA/CTS) hydrogels combined with BMSCs sheet as bone substitute in the treatment of IBDs.

\section{Methods}

The BMSCs were harvested and cultured into cell sheet. After successful establishment of animal model with chronic osteomyelitis, the 48 New Zealand white rabbits were randomly divided into 4 groups. Animals in group A were treated with thorough debridement as control. Group B were managed with BMSCs sheet. $\mathrm{CSH} / \mathrm{n}-\mathrm{HA} / \mathrm{CTS}$ hydrogels were implanted in group $\mathrm{C}$, and group $\mathrm{D}$ were treated with $\mathrm{CSH} / \mathrm{n}-\mathrm{HA} / \mathrm{CTS}+\mathrm{BMSCs}$ sheet. Gross observation and 3D reconstruction of Micro CT were performed to assess the osteogenic and infection elimination ability. Histological staining (Hematoxylin and eosin and Van Gieson) was used to observe inflammatory cell infiltration and formation of collagen fiber $4,8,12$ weeks after implantation.

\section{Results}

The bone defect of the control group was not repaired in 12 weeks as chronic osteomyelitis still existed. HE staining showed a large number of inflammatory cell infiltration around the tissue, and VG staining showed no new collagen fiber formation. In the group of BMSCs sheet, though new bone formation was observed by gross observation and Micro-CT scan, the infection was not effectively controlled due to the unfilled cavity. Some neutrophils and only a small amount of collagen fiber could be observed. Both groups of hydrogels and hydrogels/BMSCs achieved satisfactory repair effect and infection control. 3D reconstruction of Micro-CT at 4 weeks showed that the hydrogels/BMSCs sheet group had higher reconstruction efficiency and better bone modeling with normal morphology. HE staining showed little aggregation of inflammatory cells, and VG staining showed a large number of new collagen fibers.

\section{Conclusions}

Our preliminary results proved that compared with single material, the novel antibiotic-impregnated hydrogels acted as superior scaffold for BMSCs sheet and excellent antibiotics vector against infection, which provided a basis for applying tissue engineering technology to the treatment of chronic osteomyelitis. 


\section{Background}

Infected bone defects (IBDs) include both infection and defects, which are mutually causative. The sequestrum formation after infection leads to bone defects. Meanwhile, large defects are not conducive to local infection control, and the formation of hematoma in defects can become hotbed for bacterial breeding [1]. The high incidence of IBDs is due to such factors as osteomyelitis, traumatic events and iatrogenic factors [2, 3]. Effective treatment should not only eliminate infection but also repair defect, so as to alleviate the pain of patients and restore the normal function of limbs. Traditionally, the treatment strategy of IBDs can be summarized as follows: control and elimination of infected factors, maintenance of fracture stability, reconstruction of bone defects, and proper functional exercise after surgery $[4,5]$. Thorough debridement and defect reconstruction can be carried out either in one stage or two according to site, scope, and severity of infection [6].

Recently, researches on single-stage bioabsorbable bone substitutes filling to provide carriers for antibiotic releasing while repairing defects after debridement have attracted the interest of surgeons, which can overcome the limited supply of autologous bone and the risk of disease transmission of allograft bone [7-9]. Artificial synthetic bone substitutes like calcium sulfate hemihydrate (CSH) and nano-hydroxyapatite ( $\mathrm{n}-\mathrm{HA}$ ) are reasonable alternatives, as they are biodegradable and osteoconductive with excellent biocompatibility. In addition, they can be also used as the vector for antibiotic release. Although the biomechanical strength of CSH has been improved compared with CS alone, its absorption rate is still faster than the rate of new bone regeneration. n-HA has low absorption rate, while with higher solubility, stronger surface energy and greater absorbability than HA. The combination of CSH and n-HA overcome the deficiency of the two materials alone, which has synergistic osteogenic properties $[10,11]$. The introduction of organic material chitosan (CTS) makes up for the weakness that inorganic materials such as $\mathrm{Ca}^{2+}$ or $\mathrm{PO}_{4}{ }^{3-}$ can easily increase local $\mathrm{PH}[12,13]$. It not only enables the composites with the activity of regulating immunity, but also increases its stability and sustained antibiotic release efficiency as well as simulates the natural composition of the bone.

Cell sheet technology (CST) was first proposed by Japanese professor OKANO in 1993 [14]. The greatest advantage of this technique is that it does not require enzymes to acquire cultured cells, and preserves the extracellular matrix (ECM) and cell-to-cell adhesion proteins, as well as lon channels and growth factors receptors contained [15-18]. BMSCs have the potential of multidirectional differentiation and rapid proliferation, and can be directed to differentiate into chondrocytes and osteocyte. Making BMSCs into cell sheet in vitro enables it with biocompatibility and high inoculation rate, meanwhile, it also inherits the characteristics of osteogenic ability of BMSCs. Maniatopoulos, et al first reported the use of BMSCs to induce osteogenesis in vitro in 1988 [19]. Recent studies have demonstrated the feasibility of combining BMSCs sheet with HA particles to induce new bone formation in vivo [20,21].

Local antibiotic delivery system is preferred over intravenous antibiotics because of its lower cytotoxicity (including nephrotoxicity and ototoxicity) and longer maintaining of antibiotic releasing. Varies kinds of antibiotics are available when loaded with bone substitutes, and among them vancomycin tends to be 
better choice despite of its narrow antimicrobial spectrum [11,22]. Firstly, water-soluble vancomycin is dose-dependent and is effective against the most common Staphylococcus aureus in osteomyelitis, furthermore it has the least cytotoxic effect and the normal cell activity can be affected only when the local concentrations are too high, besides it's one of the few antibiotics that can inhibit bacteria released from the biofilm $[23,24]$. However, there are no studies that evaluate the validity of vancomycin-loaded $\mathrm{CSH} / \mathrm{n}-\mathrm{HA} / \mathrm{CTS}$ hydrogels combined with BMSCs sheet for the treatment of IBDs in rabbits.

In our study, the new type of vancomycin loaded CSH/n-HA/CTS hydrogels are injectable as well as biodegradable which attributed to the mechanism of cross-linking of the Schiff-base reaction between amino and aldehyde groups of carboxymethyl chitosan (CMCS) and oxidized alginate. The combination of organic and inorganic materials not only enables the hydrogels with proper mechanical properties, suitable degradation rate, osteogenesis and properties of antibiotics loading, but also preserves the extracellular matrix and growth factors of BMSCs with multi-directional differentiation potential by introducing BMSCs sheet. We applied VA/CSH/n-HA/CTS hydrogels combined with BMSCs sheet to the treatment of focal infected defect of rabbit tibia. Radiographic evaluation (3D Micro-CT reconstruction) and histological techniques are applied to detect and evaluate infection control and bone defect restoration, in order to provide theoretical support for the selection of bone substitutes and antibiotics in clinical for the treatment of focal infected bone defects.

\section{Methods}

\section{Preparation of BMSCs sheets.}

The BMSCs were isolated and obtained under sterile conditions from femur of both sides of New Zealand white rabbits of 10 -week-old, and made into single-cell suspension. Then adherent culture method was adopted. Cells were cultured in air with $5 \% \mathrm{CO}_{2}$ at $37^{\circ} \mathrm{C}$. Cells of passage 1 were digested by trypsin when $90 \%$ of cells reached confluence and the 3rd generation of high purity were obtained by the same method for subsequent experiment. To harvest BMSCs sheet, cells of passage 3 were seeded on the culture dish at a density of $9 \times 10^{4}$ cells/dish. When $75 \%$ of the cells reached confluence, DMEM medium (containing 10\% FBS, 100 mg/L L-ascorbic acid, 100 unit/ml penicillin G, 100 unit/ml streptomycin, and 2 $\mathrm{mmol} / \mathrm{l}$ L-glutamine) was used and cultured continuously for 15 days until the sheet was thick enough. The sheet was rinsed twice with PBS powdered and then scraped with a cell scraper.

To identify and detect the characteristics of BMSCs. The cells of passage 3 must meet the following criteria to be defined as BMSCs according to the rules of the mesenchymal and tissue stem cell committee of the international society for cellular therapy (ISCT), including plastic adherence in standard media, expression of specific surface maker (CD73+, CD90+, CD105+, CD45-, CD34-, CD14-, CD19-, and HLA-DR-), and in vitro differentiation potential into the osteocytes, adipocytes, and chondrocytes. Flow cytometry was used to detect specific markers of BMSCs. BMSCs of passage 3 were inoculated on culture dishes at a density of $1 \times 10^{5}$ cells $/ \mathrm{cm}^{2}$. Osteogenic induction medium $(10 \% \mathrm{FBS}, 100 \mathrm{mg} / \mathrm{L} \mathrm{L}$ ascorbic acid, 100 unit/ml penicillin G, 100 unit/ml streptomycin, 2 mmol/I L-glutamine, $10 \mathrm{mmol} / \mathrm{L}$ 
dexamethasone and $10 \mathrm{mmol} / \mathrm{L}$ sodium glycerophosphite) was used for culture at $37^{\circ} \mathrm{C}$ in a $5 \% \mathrm{CO}_{2}$ incubator. Nutrient solution was changed every 3 days, up to 21 days. Alkaline Phosphatase (ALP) staining was performed at 7, 14, 21 days after cultivation. For adipogenesis, Oil Red 0 staining was applied after cultivating with adipogenesis medium $(1 \mu \mathrm{mol} / \mathrm{L}$ dexamethasone, $10 \mathrm{mg} / \mathrm{mL}$ insulin, and $500 \mathrm{mmol} / \mathrm{L}$ 1-methyl-3-isobutyl-xanthine) for 21 days. The medium was replaced every 3 days. Results of staining were observed under a microscope and photographed afterwards.

\section{Hydrogels fabrication and characteristic detection.}

Calcium sulfate hemihydrate, nano-hydroxyapatite, and chitosan for experiment were purchased from Macklin Biochemical Co., Ltd (Shanghai, China), and the hydrogels were manufactured based on Schiff conjugate crosslinking theory. The ambient temperature was adjusted to $25^{\circ} \mathrm{C}$ to ensure successful preparation of hydrogels. Briefly, $4 \mathrm{~g}$ of carboxymethyl chitosan was slowly added to $100 \mathrm{ml}$ of purified water, and magnetic stirring was applied to prepare $4 \%(\mathrm{w} / \mathrm{v})$ carboxymethyl chitosan solution, followed by adding $30 \mathrm{mg}$ of vancomycin. Then $3.75 \mathrm{~g}$ of sodium alginate oxide was poured into $25 \mathrm{ml}$ of purified water, and placed in a constant temperature shaker until the flocculent precipitate completely disappeared, so as to prepare a $15 \%(\mathrm{w} / \mathrm{v})$ solution. Ultrasonic dispersion method was used after adding $\mathrm{CSH}$ and $6 \% \mathrm{n}-\mathrm{HA}$ complex. Thus, a uniform and stable solution of sodium alginate oxide containing $\mathrm{CSH} / \mathrm{n}-\mathrm{HA}$ was obtained. Carboxymethyl chitosan solution was mixed with sodium alginate oxide at a ratio of 4:1 (v/v). Finally, the mixture was poured into a $5 \mathrm{~mm} \times 10 \mathrm{~mm}$ cylindrical mold and placed in incubator of $37^{\circ} \mathrm{C}$ for 40 mins to obtain hydrogels, and sterilized with ethylene oxide.

Scanning electronic microscopy (SEM, Hitachi, Japan) was used to observe the microstructure and porosity of the hydrogels. Bacterial inhibition zone test: Briefly, $100 \mu \mathrm{l} \mathrm{Staphylococcus} \mathrm{aureus} \mathrm{(BeNa}$ Culture Collection, Bei Jing, China) suspension liquid at a concentration of $10^{7}-10^{8} \mathrm{CFU} / \mathrm{ml}$ was coated in Luria-Bertani (LB) solid medium evenly. Then the manufactured hydrogels were pasted on the medium and cultured at a temperature of $37^{\circ} \mathrm{C}$ with culture dish inverted. 18 hours later, bacterial growth was judged by measuring the diameter of inhibition zone with vernier calipe. Then samples were transferred to new medium containing Staphylococcus aureus for culture sequentially. The medium was changed after measuring the zone once a day until disappeared. Images were photographed digitally.

\section{Establishment of animal model.}

A total of $48 \mathrm{New}$ Zealand white rabbits provided by the experimental animal center of Air Force Medical University were included in the study, half male and half female. Each weighed between $4.5-5.5 \mathrm{~kg}$. All animal processes were approved by institutional animal care and use of Tangdu Hospital, Air Force Medical University, and was conducted in an open system laboratory with temperature of $25{ }^{\circ} \mathrm{C}$, relative humidity of about $40 \%$. The establishment of animal model of rabbit chronic osteomyelitis followed Norder method [25]. Sodium pentobarbital ( $1 \mathrm{ml} / \mathrm{kg}$, body weight) was injected into the rabbits through the ear venous. Then the rabbits were placed in a supine position on the operating table and fixed well. We selected the right tibia as the predetermined position, and after shaving, iodophor was disinfected and 
sterile tablecloths was set. We made a $3 \mathrm{~cm}$ incision along the medial side of tibia, and a $12 \mathrm{~mm} \times 6 \mathrm{~mm}$ bone window was created with electric drill and oscillating saw directly into the medullary cavity. $0.1 \mathrm{ml}$ of $5 \%$ sodium glycolate and $3 \times 10^{7} \mathrm{CFU} / \mathrm{ml}$ Staphylococcus aureus which was contained in $0.2 \mathrm{ml}$ of nutrient solution were injected to the cavity. To prevent bacterial leakage, bone wax was used to block the hole followed by irrigating with sterile saline. Rabbits were individually caged and fed with standardized fodder.

\section{Surgical procedure.}

The animal model of chronic osteomyelitis was successfully established 28 days later. Then the 48 rabbits were allocated into 4 groups according to the randomization principle. General anesthesia was applied to all operations, and we performed thorough debridement to all experiment animals. Rabbits were placed in a supine position, and the target site (right hind leg) was shaved and sterilized with iodophor. Incise the skin along the original incision until the infected defect was exposed. Eliminate the sinus tract and perform full debridement of soft tissue layer by layer. Necrotic bone was removed by laminectomy rongeur, as well as pus from the intramedullary cavity. The defect was irrigated with sterile saline 2-3 times, and was soaked in dilute iodophor saline for 5 mins after which the sterile tablecloths and gloves were replaced. In Group A $(n=12)$ we ignored the defect as blank controller. In Group B $(n=$ $12), B M S C$ s sheet was used as filler. In Group $C(n=12)$, the defect was treated with VA/CSH/n-HA/CTS hydrogels, and animals in Group D $(n=12)$ were implanted with VA/CSH/n-HA/CTS/BMSCs sheet hydrogels. The wound was sutured and analgesic was instituted after operation. Gross observation, radiographic evaluation, and histological staining were performed 4, 8, 12 weeks after operation. The operation process was as follows (Fig. 1).

\section{Gross observation.}

3 experimental animals in each group were euthanized, and gross tibial specimens were collected to assess soft tissue condition, callus formation and whether chronic osteomyelitis still existed 4,8 and 12 weeks postoperatively.

\section{Imaging measurement.}

Postoperatively, 3 rabbits in each group were selected for Micro-CT evaluation to acquire 3D images at 4, 8,12 weeks respectively. After routine anaesthesia with sodium pentobarbital ( $1 \mathrm{ml} / \mathrm{kg}$, body weight), animals were immobilized on the operating table. Tibia samples around the defect were carefully collected with the surrounding soft tissue removed, soaked in $4 \%$ paraformaldehyde. Micro-CT imaging system ( $\mu$ CT 40, Siemens, Germany) was used to evaluate infection control, hydrogels absorption and bone formation. Make sure that the long axis of the tibial sample was perpendicular to the $\mathrm{X}$-ray beam during scanning by fixing on a proper holder. The target area (Voxel size: $200 \times 80$ ) for evaluation was determined to contain no cortical bone and adhere to bone substitutes. 1,536 images were obtained from each sample and were measured by imaging experts. 3D images reconstructed obtained were analyzed using Inveon Research Workplace 2.2 software. Quantitative indicators of bone volume to total volume 
ratio (BV/TV), trabecular thickness (Tb. Th), and trabecular number (Tb. N) were collected for assessment of bone formation and material absorption.

\section{Histological evaluation.}

Hematoxylin and eosin (HE) staining was used qualitatively to detect whether inflammatory cell infiltration around tissues existed, and Van Gieson staining (VG) to observe the formation of collagen fiber. The tibia samples of each group were firstly fixed by soaking in 10\% neutral formaldehyde for 2 days. Subsequently, decalcified with $10 \%$ ethylenediaminetetraacetic acid (EDTA, pH 7.0) and dehydrated with alcohol of gradient concentration $(70 \%, 80 \%, 90 \%, 100 \%)$. Embedded in methyl methacrylate and sectioned at $10 \mu \mathrm{m}$ with microtome (Leica RM2235 microtome, Leica Microsystems, Germany) for HE staining. Another group of harvested samples that were not decalcified after fixation, were routinely dehydrated and embedded for 21 days. Sectioned with LA2500 (Leica Microsystems, Germany) at $10 \mu \mathrm{m}$ for VG staining.

\section{Statistical analysis.}

SPSS statistics software (Version 23. 0; International Business Machines Corporation, Armonk, NY, USA) was used for data analysis. All quantitative data are presented as mean \pm standard deviations and nonparametric test was performed in this study. $P$ values $<0.05$ were considered to be significant. All results were performed by statisticians unrelated to the experiment.

\section{Results}

\section{Morphological characteristics and identification of BMSCs.}

The obtained BMSCs were managed for 7 days in primary culture until $80-90 \%$ of cells reached confluence, and then received subculture. Adherent cells began to appear in 24 hours and gradually increased in 48 hours. Different multiples of optical microscope demonstrated as follows: cells of passage 1 were short spindle shaped, polygonal, and storiform with vigorous growth (Fig. 2a), while cells of passage 2 were with higher density and meaningful colonies appeared as well as stable proliferation (Fig. $2 \mathrm{~b}$ ), and the passage 3 presented as long spindle cells helically shaped and proliferated rapidly with uniform structure and high purity (Fig. 2c).

Surface antigen expression (including CD73, CD90, CD105, CD34, HLA-DR, CD14, CD45 and CD19) were detected by flow cytometry. We found the 3rd passage of cultured cells were positive for CD73 (96.19\%), CD90 (99.26\%) and CD105 (99.58\%), whose presentation rate were all over 95\%, while negative for CD34 (0.23\%), HLA-DR (0.77\%), CD14 (2.8\%), CD45 (0.17\%) and CD19 (4.4\%), which confirmed that the 3rd generation of cultured cells were BMSCs rather than hematopoietic stem cell. (Fig. 3)

14 days after culture of BMSCs (Passage 3), a white translucent sheet-like substance with certain thickness could be found at the bottom of the petri dish, which had certain mechanical strength and 
elasticity and could be curled or folded. The cell sheet could be gently peeled off with sterilized ophthalmic forceps (Fig. 4a). After 14 days of osteogenic induction of BMSCs (Passage 3), ALP staining showed positive with the cytoplasm presenting blue and black (Fig. 4b). After 21 days of induction, Alizarin Red staining showed typical mineralized nodule (Fig. 4c), and Oil Red 0 staining of lipogenetic differentiated BMSCs showed the lipid droplets in the cytoplasm were stained with orange red (Fig. 4d).

\section{Characteristics of VA/CSH/n-HA/CTS hydrogels.}

Figure 5a presented intuitively the white hydrogels scaffold after synthesis, showing that it is injectable with good fluidity. The hydrogels were lyophilized into cylindrical shape for sectioning by a specific mold only for observation of the microstructure with scanning electron microscopy (SEM) (Fig. 5b). When the manufactured hydrogels were pasted on the medium and cultured at a temperature of $37^{\circ} \mathrm{C}$ with culture dish inverted for 18 hours, we could see an obvious inhibition zone for staphylococcus aureus with an average diameter of $15 \mathrm{~mm}$, indicating that the gel had a good inhibition effect on SA (Fig. 5c).

SEM micromorphology of hydrogels with different materials after freeze-drying microtomy (Fig. 6). Generally, the scaffold materials still maintained a continuous three-dimensional network structure at low magnification (300x), meanwhile the pores were interpenetrating with good connectivity, and the pore size was 80-200 $\mu \mathrm{m}$, with an average of $140 \mu \mathrm{m}$. (Fig. 6a, b). With the introduce of the intensifying phase and vancomycin, the order and size of the pore also decreased, transforming to disorderly from the original regular arrangement gradually, indicating that the introduce of $\mathrm{CSH} / \mathrm{n}-\mathrm{HA}$ and vancomycin also promoted the cross-linking of hydrogels, which resulted in a more compact network structure, besides it could be observed that the hydrogels had a more porous structure due to the wide distribution of VA, and this right-sized aperture also provided proper space for new bone to grow in. (Fig. 6c). At the same time, due to the addition of $\mathrm{n}-\mathrm{HA}$ and $\mathrm{CSH}$, mineral crystallization deposition could be observed in the pore of the composite scaffold, which appeared very rough (Fig. 6b). Higher porosity contributed to BMSCs ingrowth and vascularization.

\section{Successful establishment of animal models.}

4 weeks after the preparation of chronic osteomyelitis model of the rabbits, all the wound of models produced pus (Fig. 7a, b), with local soft tissue swelling and lameness of the injured limb, accompanied by various extents of anorexia and weight loss. Bacterial cultures showed positive of staphylococcus aureus (Fig. 7c). 3 cases died because of bacteremia 2 weeks postoperatively, while the rest of the models in radiology are characterized by the typical osteomyelitis 4 weeks later, gross observation showed sinus tract formation (Fig. 7d). Radiographic examination revealed moderate to extensive cortical reaction, cortical bone destruction and new bone formation (Fig. 7e, f). Osteomyelitic sequesetration could be observed in the proximal tibial medullary cavity, and pathological examination revealed chronic active inflammation with bone dissolving and new fibrous bone formation (Fig. $7 \mathrm{~g}$ ).

Gross observation. The right tibia samples of the four groups were harvested for gross observation at 4 weeks, 8 weeks and 12 weeks after thorough debridement and planned treatment. Control group showed 
typical osteomyelitis and severe tibia damage at 4 weeks and 8 weeks. and the bone defect remained unhealed with deformity of the tibia at 12 weeks. In group of BMSCs sheet, the defect appeared to be as obvious as originally, but over time, it was partially healed although the infection was uncontrolled. In group $\mathrm{C}$ treated with $\mathrm{VA} / \mathrm{CSH} / \mathrm{n}-\mathrm{HA} / \mathrm{CTS}$, infection was eliminated and the defect restored much more completely than in group B. In group D treated with VA/CSH/n-HA/CTS/ BMSCs sheet, it could be seen that at first, a clear boundary existed between hydrogels and the bone tissue. As the absorption of bone substitutes, abundant fibrous tissue was formed, and the defect cavity gradually decreased with novel bone forming, meanwhile infection was effectively controlled. The defect was almost completely reconstructed 12 weeks postoperatively.

3D reconstruction of Micro-CT evaluation. Micro-CT scans were obtained at 4 weeks, 8 weeks, and 12 weeks postoperatively. On the whole, it demonstrated that in group A, the defect still existed with almost no new born formation, while in group $B$ callus formed with a density similar to that of the surrounding bone tissue and the defect cavity was partly treated. In group $C$ and $D$, the hydrogels were gradually absorbed over time and the new bone formation was almost complete at 12 weeks. Meanwhile, by 3D reconstruction of the target position, material degradation, new bone formation in each group were assessed and evaluated quantitatively (Fig. 10). 4 weeks after surgery, distinct cavities could be noticed in all groups, and callus formation was observed in group $B, C$, and $D$. In general, the BV/TV, Tb. N, Tb. Th of the four groups showed significant difference with $P \otimes 0.05(P=0.016, P=0.016, P=0.016)$, while only BV/TV was statistically significant between groups. Statistical results showed that at 4 weeks, the BV/TV of the experimental group was better than the other three groups, especially better than group C. At time point of 8 weeks, though the defect of the control group and the BMSCs sheet group tended to shrink, it showed typical radiographic symptoms of chronic osteomyelitis. As the material degraded, most of the defects were repaired and filled with novel bone in the VA/CSH/n-HA/CTS and VA/CSH/n$\mathrm{HA} / \mathrm{CTS} / \mathrm{BMSC}$ sheet group with no recurrence of infection. Statistics analysis showed in general there was difference in BV/TV, Tb. $N$ of the four groups $(P=0.030, P=0.016)$, and the BV/TV of Group $D$ was statistically different from Group A and B, while no different from Group C. At 12 weeks postoperatively, chronic osteomyelitis still existed in control and BMSCs sheet group with cavity, rim osteosclerosis and ossification. All material degraded and novel bones filled the defect completely in VA/CSH/n-HA/CTS and VA/CSH/n-HA/CTS/BMSCs sheet group, as was shown in Fig. 10. In general, the BV/TV of the four groups showed significant difference with $\mathrm{P}=0.016$, while $\mathrm{Tb} . \mathrm{N}$ and $\mathrm{Tb}$. Th of the four groups showed no difference $(P=0.055, P=0.218)$. Meanwhile, there was no statistical difference in new bone formation among groups of $B, C$, and $D$.

Van Gieson staining. 4 weeks postoperatively, the VG staining images showed clear boundary of the bone formation area in the four groups (Fig. 11). No new collagen fiber formed in Group A, and a small amount of collagen fiber formed in Group B, C and D. Hydrogels were partially absorbed in Group C and D, with sharp interface between bone tissue. At 8 weeks, compared with Group A and B, Group C and D showed a number of collagen fibers, accompanied by defect decreases and novel bone formation. While 12 weeks postoperatively, defects were still visible in Group A, which contained only a small amount of collagen 
fibers. Defects were partially healed in Group B, with bone in disorders. Defects were absolutely reconstructed in Group C and D, of which we could see rich collagen fibers in group D.

Hematoxylin and Eosin staining. The HE staining was performed to identify the characteristics of the experimental sites of the four groups to further evaluate the contribution of different factors to the infection control. Plentiful inflammatory cells were observed in both the control group and Group B, indicating that infection was not effectively eliminated. While in $\mathrm{VA} / \mathrm{CSH} / \mathrm{n}-\mathrm{HA} / \mathrm{CTS}$ and $\mathrm{VA} / \mathrm{CSH} / \mathrm{n}$ HA/CTS/BMSCs sheet group, inflammatory cells decreased gradually over time, suggesting that the antibiotic loading system was effective in treating infection (Fig. 12).

\section{Discussion}

Thorough debridement is the fundamental of effective therapy for chronic osteomyelitis [26, 27]. However, defects lacking of vascularization make systemic antibiotic curative effect unsatisfactory, with high recurrence of infections. In order to deal with defect, autologous or allogeneic bone are traditionally chosen, while problems such as limited source, complications of donor site and disease transmission remain insurmountable [28]. Thus, the demand for synthetic scaffold material for bone tissue engineering is increasing for years as they offer a more promising strategy to overcome the conundrum of treating IBDs that plagues orthopaedic surgeons [2]. It has been reported that the combination of appropriate inorganic materials and BMSCs may provide potential for bone regeneration [29]. The ideal injectable bone tissue engineering materials should meet the following characteristics: excellent biocompatibility and biodegradability; capacity of bone induction and bone conduction; proper in vivo degradation rate that matches with the pace of osteogenesis; appropriate structure and porosity for the ingrowth of blood vessels and cell distribution [30, 31].

The BMSCs sheet technology overcomes the problem of low osteogenic ability of BMSCs suspensions by maintaining ECM and cell to cell communication, and since its development, the sheet technology has been successfully applied in soft tissue reconstruction, bone defect repair and other fields [32, 33]. The reasonableness that we chose $\mathrm{CSH} / \mathrm{n}-\mathrm{HA} / \mathrm{CTS}$ as scaffold to carry BMSCs sheet in our research could be expounded as following: It has good biomechanical properties and compatibility, which has been confirmed by previous studies, moreover, Cabañas MV, et al and Frame JW have shown in their study that the mix of $\mathrm{CSH}$ and $\mathrm{n}-\mathrm{HA}$ improved the poor injection performance of single material [34-37]. In addition, chitosan possesses ability of inhibitory effect of microorganisms, activation and chemotaxis effect of macrophages $[38,39]$. When combined with synthetic bone substitutes, chitosan provide space for the growth of new bone and vessels, and with the change of surrounding temperature, chitosan can be transformed from liquid phase to solid phase [40].

Therefore, the combination of CSH/n-HA/CTS hydrogels, which has the ability of vancomycin releasing accompanied with promoting osteogenesis, and BMSCs sheet rich in ECM as well as various growth factors tend to be a choice for treatment of IBDs caused by chronic osteomyelitis, which may be an ideal method to repair the defect and control the infection effectively. 
In our study, infection was effectively controlled in CSH/n-HA/CTS group and CSH/n-HA/CTS/BMSCs sheet group during 12 weeks follow-up, while chronic osteomyelitis still existed in control group and BMSCs sheet group which was also verified by HE staining. Analyzing the reasons, we believed that on the premise of effective debridement, the presence of persistent cavity was not conducive to elimination of infection. Despite the application of BMSCs sheet in group B, which enables bone formation, but as it couldn't provide continuous defect filling effect and physical support characteristics, leading to the presence of infection. By contrast, due to the introduce of $\mathrm{CSH} / \mathrm{n}-\mathrm{HA} / \mathrm{CTS}$ hydrogels in group $\mathrm{C}$ and $\mathrm{D}$, the absorption and degradation rate of the material was suitable, so that new defects caused by excessive absorption would not be generated. Through clinical research, Studies showed that the selection of bone substitutes with appropriate degradation rate played a significant role in the treatment of chronic osteomyelitis [41]. At the same time, the capacity of the antibiotic loading of CSH enabled the local area to maintain a high concentration of antibiotics for a long time. Effective infection control provided a prerequisite for defect reconstruction. Besides, vancomycin was selected as local releasing antibiotic because of its effectiveness against the common pathogenic bacteria of osteomyelitis (MSSA and MRSA) and its low cytotoxicity to the surrounding tissues [42]. Studies on local drug concentration release curve of vancomycin showed that the concentration dropped slowly and lasted for a long period [43].

Through 3D Micro-CT reconstruction, we found that sufficient new bone formed to repair the defect in both group $C$ and $D$, which had significant difference with the other two groups at 4 weeks. Meanwhile, the capacity of bone formation at 4 weeks in group $D$ was better than that in group $C$, that had also been proved by quantitative analysis, however, as time went on to 8 and 12 weeks, there was no difference in bone formation between the two groups, indicating that the application of BMSCs sheet in group D might promote the rate of osteogenesis within 4 weeks. But further study is still needed. What's interesting in the control group is that the amount of new bone formation increased from week 4 to week 8 , while decreased from week 8 to week 12. By analyzing the reasons, we believed that at 8 weeks due to the persistence of infection, partial inflammatory callus formed, and up to 12 weeks, a large amount of osteonecrosis led to a reduction in new bone formation during quantitative calculation of 3D reconstruction.

VG staining showed that the content of collagen fiber in group D was higher at the same period, and it was closely crosslinked with the scaffold material. However, over time up to 12 weeks, there was no difference in bone mass between the group $\mathrm{C}$ and $\mathrm{D}$. The high porosity of hydrogels provided plenty of space for ingrowth of new bone and blood vessels and surface for direct cell adhesion and proliferation [44]. Besides, observation by gross anatomy, we found that the composite material partly degradation in about 4 weeks, and almost entirely in 8 weeks. Reasons could be analyzed and associated with the introduce of $\mathrm{CSH}$, as reported that degradation rate of $\mathrm{CSH}$ varied from 1.5 to 3 months in vivo and ratio of $\mathrm{CSH}$ and n-HA directly affect the structure of the hydrogels as well as degradation speed, which tends to be the trench for our future research $[11,45]$. The technique of locally minimally invasive BMSCs acquisition, separation and cultivation into sheet in vitro would significantly reduce the demand for autologous bone which could cause certain collateral damage. 
However, our study has some shortcomings. Our study was designed for the reconstruction of focal IBDs, without considering the treatment of segmental IBDs, and lacked real-time monitoring and evaluation of the degradation of synthetic hydrogels in vivo, which would help us optimize the ratio of different materials in hydrogels so as to improve its performance. Meanwhile, the biomechanical analysis of hydrogels, local drug elution kinetics monitoring and pharmacokinetics characteristics after loaded with vancomycin still need to be explored. In addition, hydrogels combined with BMSCs should also be set as a control group, and further validation in large animal models (sheep or pigs) is the prerequisite for preclinical study in the future.

\section{Conclusion}

This technique of combining organic (CTS and BMSCs sheet) and inorganic materials (CSH/n-HA/CTS) not only overcame the drawbacks of single material, but gained proper osteogenesis ability. Meanwhile it showed excellent biocompatibility and biodegradability in the process of bone defects reconstruction of IBDs. The introduction of CSH equipped the hydrogels with characteristics of antibiotics releasing, which played an irreplaceable role in the elimination of infection and the addition of BMSCs sheet may accelerate the rate of bone formation. The VA-loaded hydrogels, as bone substitutes and drug delivery carrier, provide a new idea for the therapy of IBDs.

\section{Abbreviations}

IBDs

Infected bone defects

$\mathrm{CSH}$

Calcium sulfate hemihydrate

$\mathrm{n}-\mathrm{HA}$

nano-hydroxyapatite

CTS

Chitosan

BMSCs

Bone marrow mesenchyml stem cells

$\mathrm{HE}$

Hematoxylin and eosin

VG

Van Gieson

ECM

Extracellular matrix

CMCS

Carboxymethyl chitosan

ALP 
Alkaline phosphatase

SEM

Scanning electron microscopy.

\section{Declarations}

\section{Ethics approval and consent to participate}

Our study received permissions from the ethics committee of Tang Du Hospital, Air Force Medical University, and was performed with their guidance and help.

\section{Consent for publication}

Not applicable.

\section{Competing interests}

The authors declare that they have no competing interest.

\section{Author details}

${ }^{1}$ Department of Orthopaedics, Second affiliated hospital, Air Force Medical University, Xi'an, Shaanxi, 710038, China; ${ }^{2}$ Department of Ultrasound, Xijing Hospital, Air Force Medical University, Xi'an, Shaanxi 710032, P.R. China; ${ }^{3}$ Institute of Oral Tissue Engineering, Air Force Medical University, Xi'an, Shaanxi 710032, P.R. China.

\section{References}

1. Aufricht G. Dental molding compound cast and adhesive strapping in rhinoplastic surgical procedure. Arch Otolaryngol. 1940;32(2):333-8.

2. Pearson JJ, Gerken N, Bae C, Lee KB, Satsangi A, McBride S, et al. In vivo hydroxyapatite scaffold performance in infected bone defects. J Biomed Mater Res. 2020;108(3):1157-66.

3. Malkawi H, Shannak A, Sunna' P. Active treatment of segmental defects of long bones with established infection. A prospective study. Clin Orthop Relat Res. 1984;184:241-8.

4. Haas DW, McAndrew MP. Bacterial osteomyelitis in adults: evolving considerations in diagnosis and treatment. Am J Med. 1996;101(5):550-61.

5. Corona PS, Altayó M, Amat C, Vicente M, Velez R. Reconstruction of infected post-traumatic bone defects of the distal femur with the Compress implant. Preliminary results of a staged non-biological strategy. Injury. 2021;52(3):606-15.

6. Xiao H, Wang S, Wang F, Dong S, Shen J, Xie Z. Locking compression plate as an external fixator for the treatment of tibia infected bone defects. Z Orthop Unfall. 2021; doi:10.1055/a-1545-5363. 
7. Zhao ZH, Wang GL, Zhang Y, Luo W, Liu SY, Zeng ZH, et al. Induced membrane technique combined with antibiotic-loaded calcium sulfate-calcium phosphate composite as bone graft expander for the treatment of large infected bone defects: preliminary results of 12 cases. Ann Transl Med. 2020;8(17):1081-94.

8. Kimna C, Deger S, Tamburaci S, Tihminlioglu F. Chitosan/montmorillonite composite nanospheres for sustained antibiotic delivery at post-implantation bone infection treatment. Biomed Mater. 2019;14(4):044101.

9. Betz RR. Limitations of autograft and allograft: new synthetic solutions. Orthopedics. 2002;25 Suppl $5: 561-70$.

10. Jiang $Y$, Qin HJ, Wan HY, Yang J, Yu Q, Jiang M, et al. Asprin-loaded strontium-containing a-calcium sulphate hemihydrate/nano-hydroxyapatite composite promotes regeneration of critical bone defects. J Cell Mol Med. 2020;24(23):13690-702.

11. Kelly CM, Wilkins RM, Gitelis S, Hartjen C, Watson JT, Kim PT. The use of a surgical grade calcium sulfate as a bone graft substitute: results of a multicenter trial. Clin Orthop Relat Res. 2001;382:4250.

12. Boukari Y, Qutachi O, Scurr DJ, Morris AP, Doughty SW, Billa N. A dual-application poly (dl-lactic-coglycolic) acid (PLGA)-chitosan composite scaffold for potential use in bone tissue engineering. $J$ Biomater Sci Polym Ed. 2017;28(16):1966-83.

13. Shamekhi MA, Rabiee A, Mirzadeh H, Mahdavi H, Mohebbi-Kalhori D, Eslaminejad MB. Fabrication and characterization of hydrothermal cross-linked chitosan porous scaffolds for cartilage tissue engineering applications. Mater Sci Eng C Mater Biol Appl. 2017;80:532-42.

14. Okano T, Yamada N, Sakai H, Sakurai Y. A novel recovery system for cultured cells using plasmatreated polystyrene dishes grafted with poly (N-isopropylacrylamide). J Biomed Mater Res. 1993;27(10):1243-51.

15. Lu Y, Zhang W, Wang J, Yang GZ, Yin S, Tang TT, et al. Recent advances in cell sheet technology for bone and cartilage regeneration: from preparation to application. Int J Oral Sci. 2019;11(2):1-13.

16. Ito M, Shichinohe H, Houkin K, Kuroda S. Application of cell sheet technology to bone marrow stromal cell transplantation for rat brain infarct. J Tissue Eng Regen Med. 2017;11(2):375-81.

17. Xu M, Li J, Liu X, Long SQ, Shen Y, Li Q, et al. Fabrication of vascularized and scaffold-free bone tissue using endothelial and osteogenic cells differentiated from bone marrow derived mesenchymal stem cells. Tissue Cell. 2019;61:21-9.

18. Kobayashi J, Akiyama Y, Yamato M, Shimizu T, Okano T. Design of temperature-responsive cell culture surfaces for cell sheet-based regenerative therapy and 3D tissue fabrication. Adv Exp Med Biol. 2018;1078:371-93.

19. Manialopoulos C, Scdek J, Meleher AH. Bone formation in vitro by stromal cells obtained from bone marrow of young adult rats. Cell Tissue Res. 1988;254(2):317-30.

20. Deng LZ, Liu Y, Yang LQ, Yi JZ, Deng FL, Zhang LM. Injectable and bioactive methylcellulose hydrogel carrying bone mesenchymal stem cells as a filler for critical-size defects with enhanced 
bone regeneration. Colloids Surf B Biointerfaces. 2020;194:111159.

21. Naudot M, Garcia Garcia A, Jankovsky N, Barre A, Zabijak L, Zakaria Azdad S, et al. The combination of a poly-caprolactone/nano-hydroxyapatite honeycomb scaffold and mesenchymal stem cells promotes bone regeneration in rat calvarial defects. J Tissue Eng Regen Med. 2020;14(11):1570-80.

22. Walsh WR, Chapman-Sheath PJ, Cain S, Debes J, Bruce WJM, Svehla MJ, et al. A resorbable porous ceramic composite bone graft substitute in a rabbit metaphyseal defect model. J Orthop Res. 2003;21(4):655-61.

23. Fang B, Qiu P, Xia C, Cai D, Zhao CC, Chen Y, et al. Extracellular matrix scaffold crosslinked with vancomycin for multifunctional antibacterial bone infection therapy. Biomaterials. 2021;268:120603.

24. Broussou DC, Lacroix MZ, Toutain PL, Woehrlé F, Garch FE, Bousquet-Melou A, et al. Differential activity of the combination of vancomycin and amikacin on planktonic vs biofilm-growing Staphylococcus aureus bacteria in a hollow fiber infection model. Front Microbiol. 2018;9:572-82.

25. Norden CW, Kennedy E. Experimental osteomyelitis. I. A description of the model. J Infect Dis. 1970;122(5):410-8.

26. Drampalos E, Mohammad HR, Pillai A. Augmented debridement for implant related chronic osteomyelitis with an absorbable, gentamycin loaded calcium sulfate/hydroxyapatite biocomposite. J Orthop. 2020;17:173-9.

27. Bibbo C, Stough JD. Reduction calcaneoplasty and local muscle rotation flap as a salvage option for calcaneal osteomyelitis with soft tissue defect. J Foot Ankle Surg. 2012;51(3):375-8.

28. Ueha T, Akahane M, Shimizu T, Uchihara Y, Morita, Y, Nitta N, et al. Utility of tricalcium phosphate and osteogenic matrix cell sheet constructs for bone defect reconstruction. World J Stem Cells. 2015;7(5):873-82.

29. Liang J, Li W, Zhuang N, Wen SN, Huang SJ, Lu WZ, et al. Experimental study on bone defect repair by BMSCs combined with a light-sensitive material: g- $\mathrm{C}_{3} \mathrm{~N}_{4} / \mathrm{rGO}$. J Biomater Sci Polym Ed. 2021;32(2):248-65.

30. Gupta P, Adhikary M, M JC, Kumar M, Bhardwaj N, Mandal BB, et al. Biomimetic, osteoconductive non-mulberry silk fiber reinforced tricomposite scaffolds for bone tissue engineering. ACS Appl Mater Interfaces. 2016;8(45):30797-810.

31. Camarero-Espinosa S, Rothen-Rutishauser B, Weder C, Foster EJ, et al. Directed cell growth in multizonal scaffolds for cartilage tissue engineering. Biomaterials. 2016;74:42-52.

32. Tang Z, Okano T. Recent development of temperature-responsive surfaces and their application for cell sheet engineering. Regen Biomater. 2014;1(1):91-102.

33. Ma D, Ren L, Chen F, Liu Y, Zhang J, Xue Z, et al. Reconstruction of rabbit critical-size calvarial defects using autologous bone marrow stromal cell sheets. Ann Plast Surg. 2010;65(2):259-65.

34. Nilsson M, Wang JS, Wielanek L, Tanner KE, Lidgren L. Biodegradation and biocompatability of a calcium sulphate-hydroxyapatite bone substitute. J Bone Joint Surg Br. 2004;86(1):120-5. 
35. Delloye C, Cnockaert N, Cornu O. Bone substitutes in 2003: an overview. Acta Orthop Belg. 2003;69(1):1-8.

36. Frame JW, Rout PGJ, Browne RM. Ridge augmentation using solid and porous hydroxylapatite particles with and without autogenous bone or plaster. J Oral Maxillofac Surg. 1987;45(9):771-7.

37. Cabanas MV, Rodriguez-Lorenzo LM, Vallet-Regi M. Setting behavior and in vitro bioactivity of hydroxyapatite/calcium sulfate cements. Chem Mater. 2002;14(8):3550-5.

38. Patel VR, Amiji MM. Preparation and characterization of freeze-dried chitosan-poly (ethylene oxide) hydrogels for site-specific antibiotic delivery in the stomach. Pharm Res. 1996;13(4):588-93.

39. Noel SP, Courtney H, Bumgardner JD, Haggard WO. Chitosan films: a potential local drug delivery system for antibiotics. Clin Orthop Relat Res. 2008;466(6):1377-82.

40. Yan Y, Zhang X, Li C, Huang Y, Ding Q, Pang X. Preparation and characterization of chitosansilver/hydroxyapatite composite coatings on $\mathrm{TiO}_{2}$ nanotube for biomedical applications. Appl Surf Sci. 2015;332:62-69.

41. Zhao ZH, Wang GL, Zhang Y, Luo W, Liu SY, Liu YY, et al. The effect of calcium sulfate/calcium phosphate composite for the treatment of chronic osteomyelitis compared with calcium sulfate. Ann Palliat Med. 2020;9(4):1821-33.

42. Colding-Rasmussen T, Horstmann P, Petersen MM, Hettwer W. Antibiotic elution characteristics and pharmacokinetics of gentamicin and vancomycin from a mineral antibiotic carrier: an in vivo evaluation of 32 clinical cases. J Bone Jt Infect. 2018;3(4):234-40.

43. Nugent M, McLaren A, Vernon B, McLemore R. Strength of antimicrobial bone cement decreases with increased poragen fraction. Clin Orthop Relat Res. 2010;468(8):2101-6.

44. Ma PX. Scaffolds for tissue fabrication. Mater today. 2004;7(5):30-40.

45. Roberts TT, Rosenbaum AJ. Bone grafts, bone substitutes and orthobiologics: the bridge between basic science and clinical advancements in fracture healing. Organogenesis. 2012;8(4):114-24.

\section{Figures}




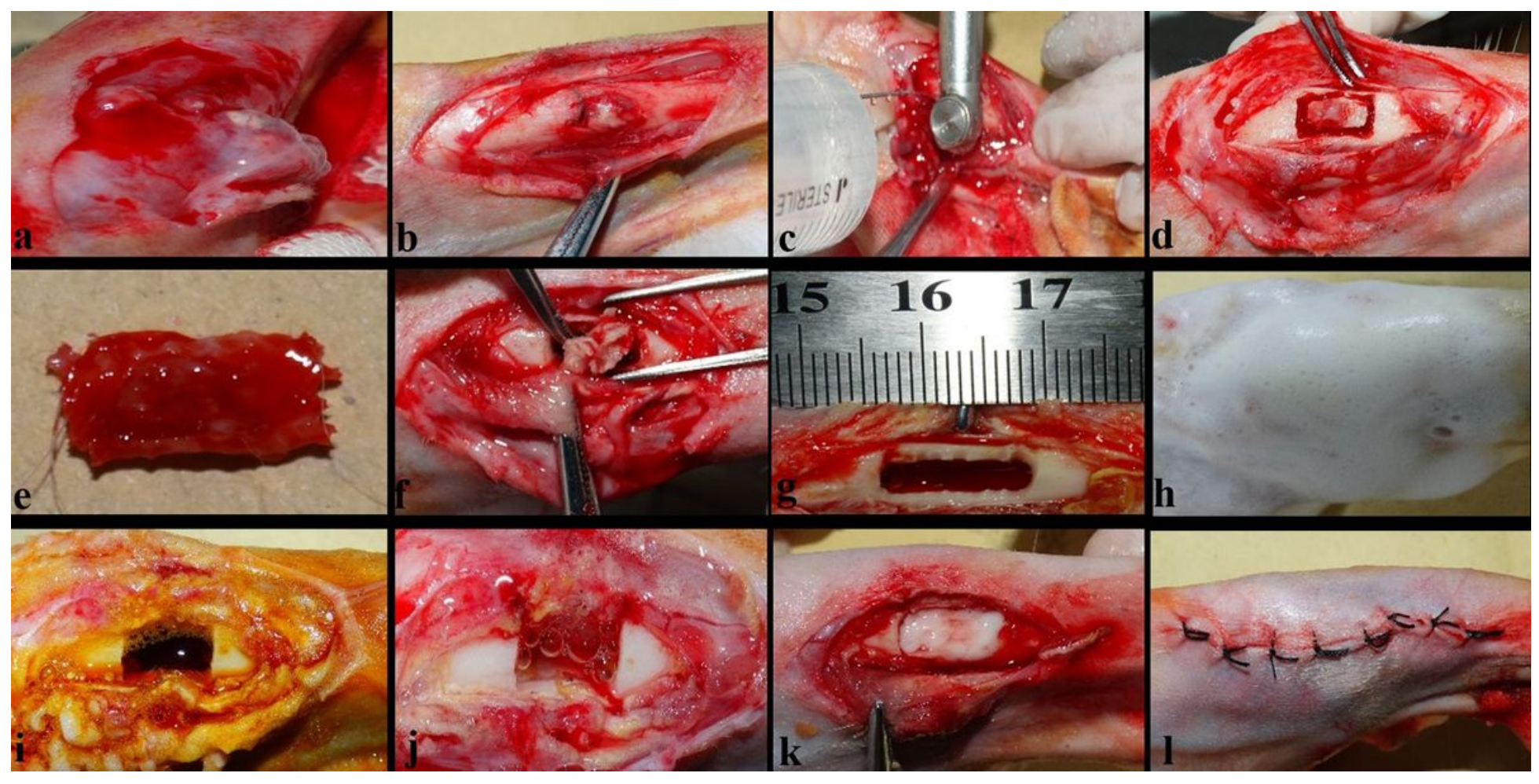

Figure 1

The surgical procedure of thorough debridement and filling of hydrogels after successful establishment of animal model. Sinus tract and infected soft tissues were eliminated layer by layer and necrotic bone was removed by laminectomy rongeur (a-e). The bone marrow cavity was thoroughly cleaned (f), after which size of the defect was measured (g). Irrigating with hydrogen peroxide, iodophor and sterile saline for 3 times $(h-j)$, then, corresponding materials were filled and the incision was sutured $(k, l)$.

\section{Figure 2}

morphology of cultured BMSCs cells under different multiples microscope. Cells were cultured for 7 days and were short spindle shaped, polygonal, and storiform with vigorous growth (a). After primary culture (the 2nd passage), adherent cells began to appear in 24 hours and gradually increased in 48 hours with higher density and meaningful colonies (b). After passage culture (the 3rd passage) of 7 days, cells presented as long spindle helically shaped and proliferated rapidly with uniform structure and high purity (c). 
CD73

CD90

CD105

CD34
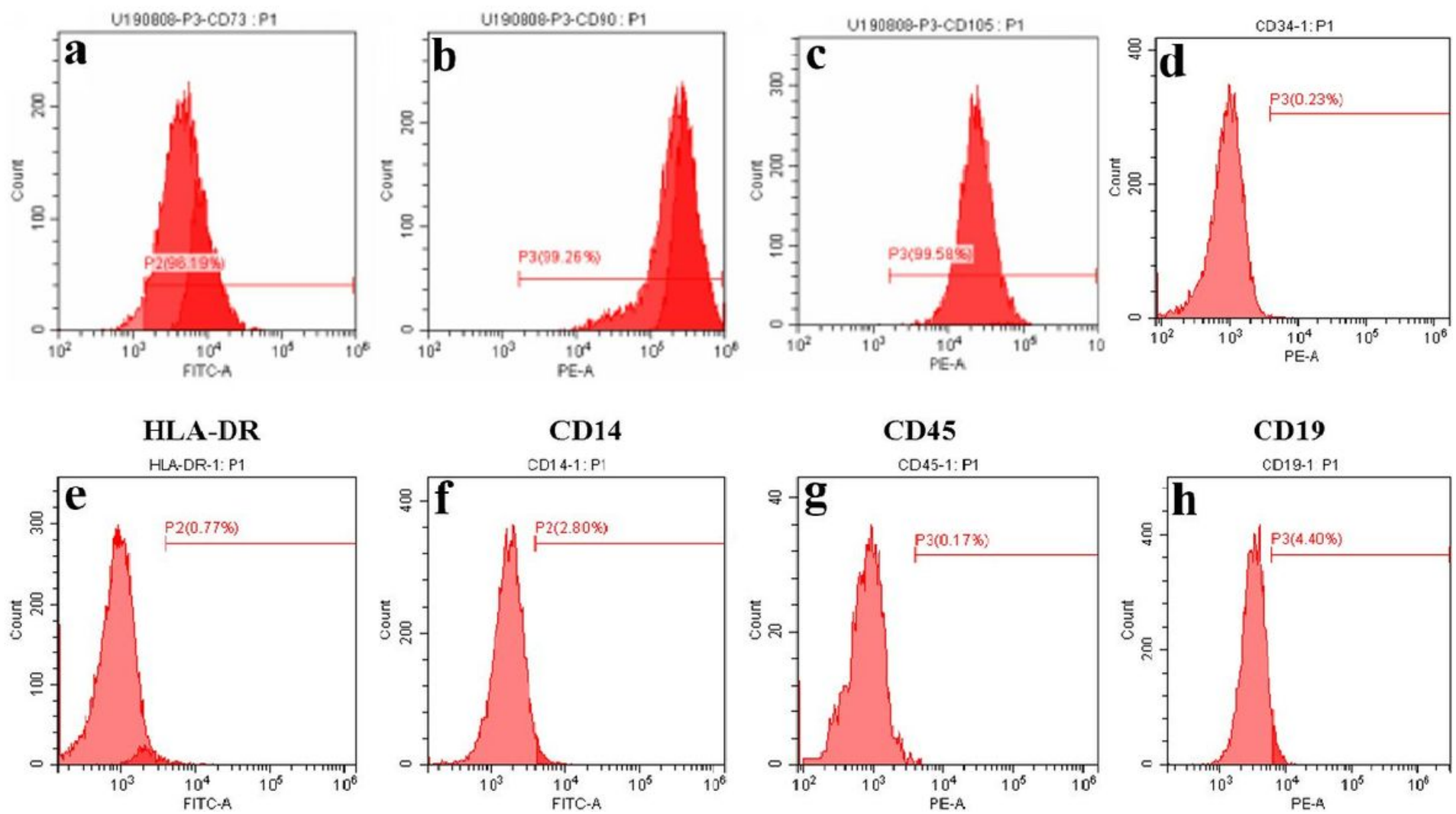

Figure 3

Results of flow cytometry detection of BMSCs (the 3rd passage). a. CD73 (+); b. CD90 (+); c. CD105 (+); d. CD34 (-); e. HLA-DR (-); f. CD14 (-); g. CD45 (-); h. CD19 (-).

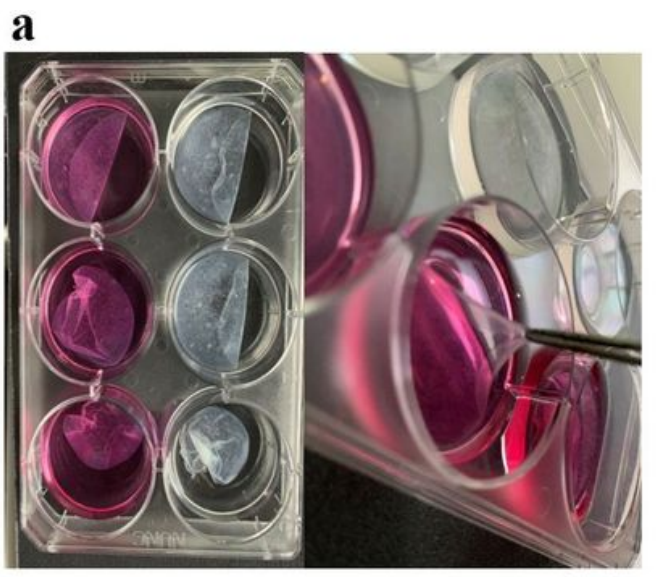

BMSCs Cell Sheet b

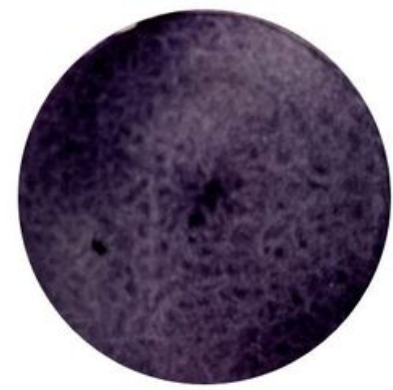

ALP staining c

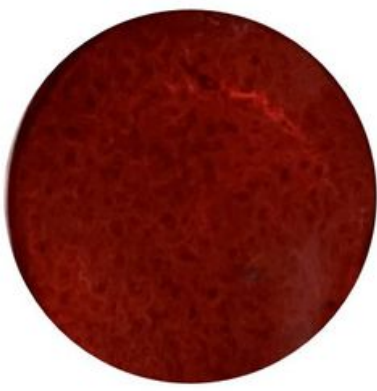

Alizarin Red staining d

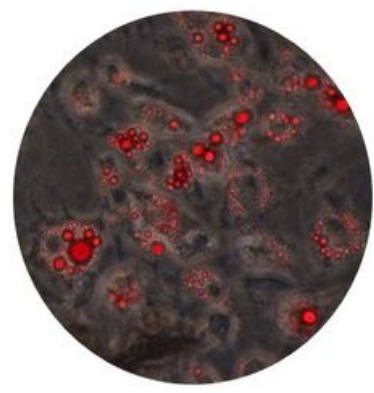

Oil Red O staining

Figure 4

Cell sheet was achieved 14 days after culture in vitro of BMSCs (passage 3). It was translucent with certain thickness and had certain mechanical strength and elasticity (a). Alkaline phosphatase (ALP) staining of BMSCs after osteogenic induction showed positive with the cytoplasm presenting blue and 
black (b), Alizarin Red staining showed typical mineralized nodule with orange red (c), and lipid droplets stained with Oil Red 0 staining showed orange red under microscope (d).

a

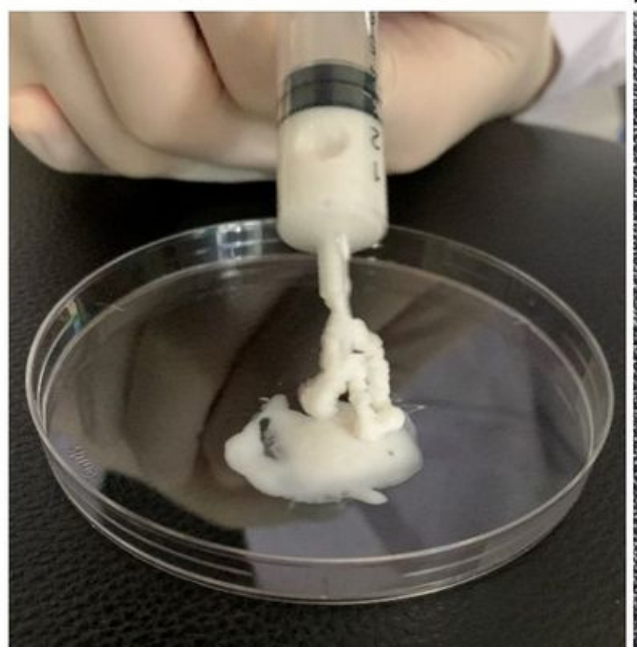

b

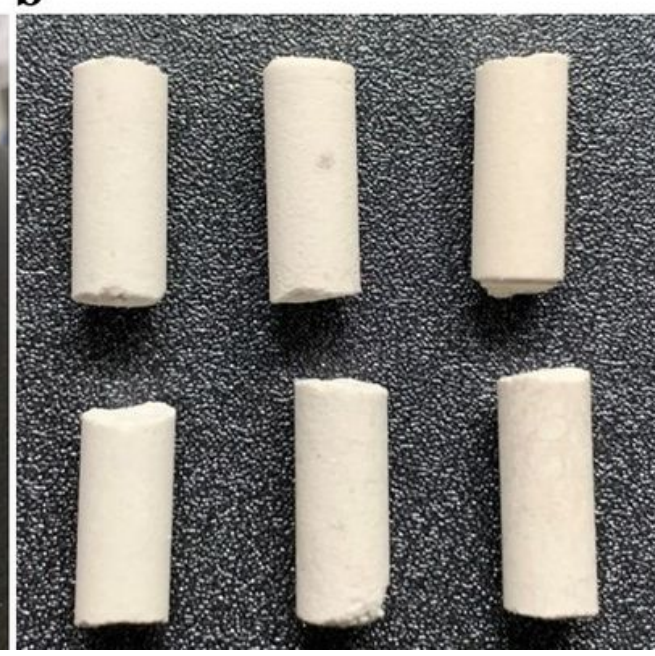

c

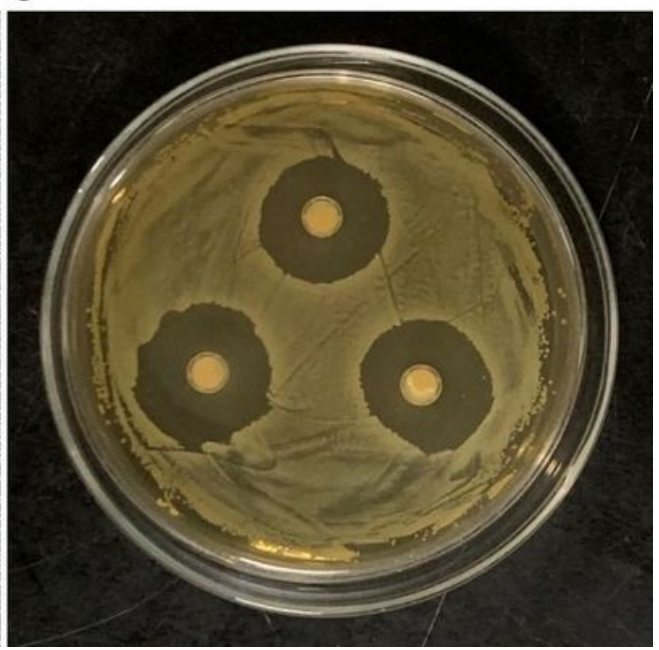

Figure 5

Characteristics of synthetic VA/CSH/n-HA/CTS hydrogel. It was injectable with good fluidity and was lyophilized into cylindrical shape for observation with SEM $(a, b)$. Inhibition zone for staphylococcus aureus with an average diameter of $15 \mathrm{~mm}$ could be observed (c).

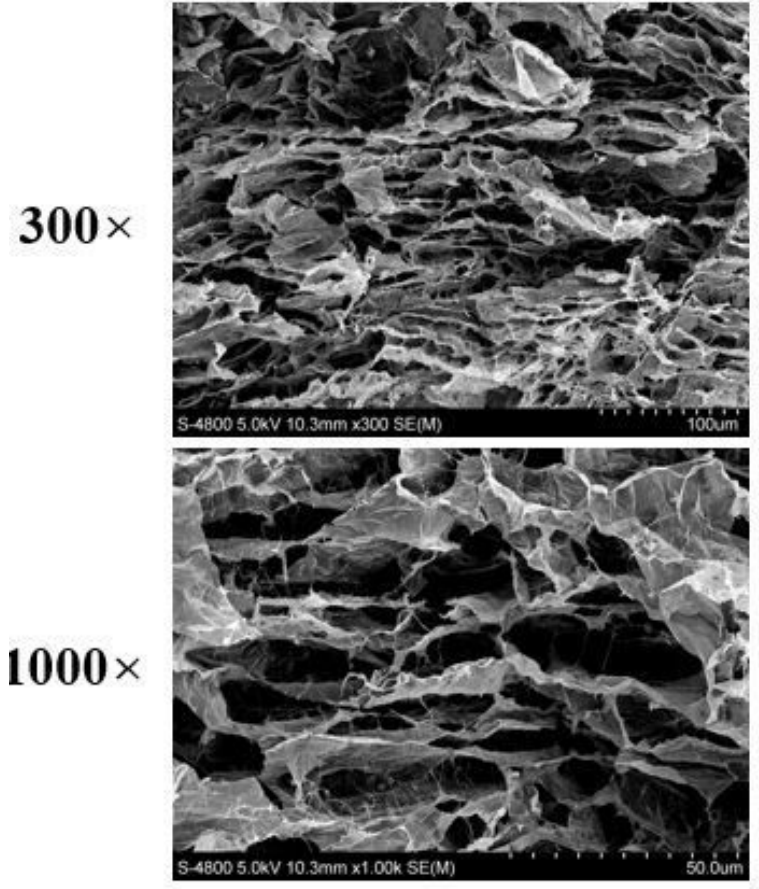

a. CTS

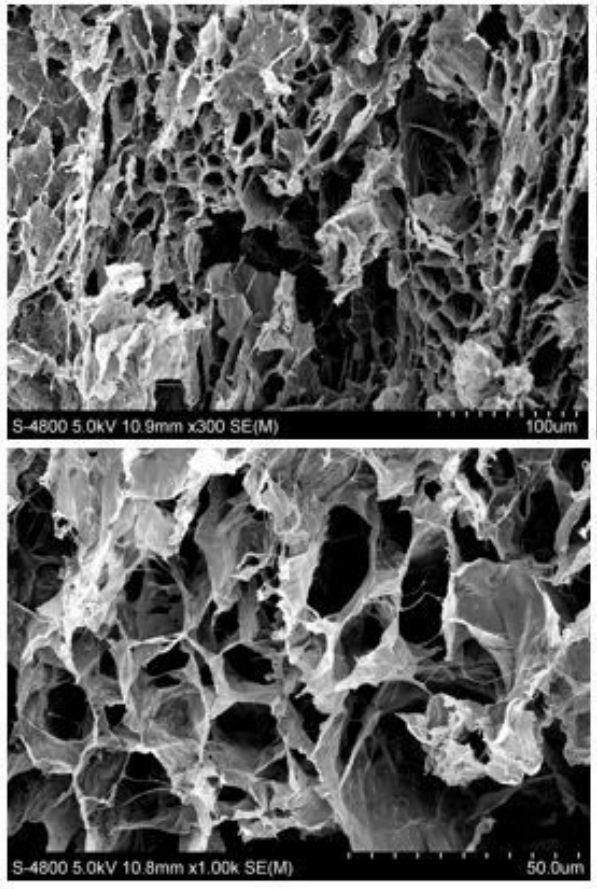

b. CSH/n-HA/CTS

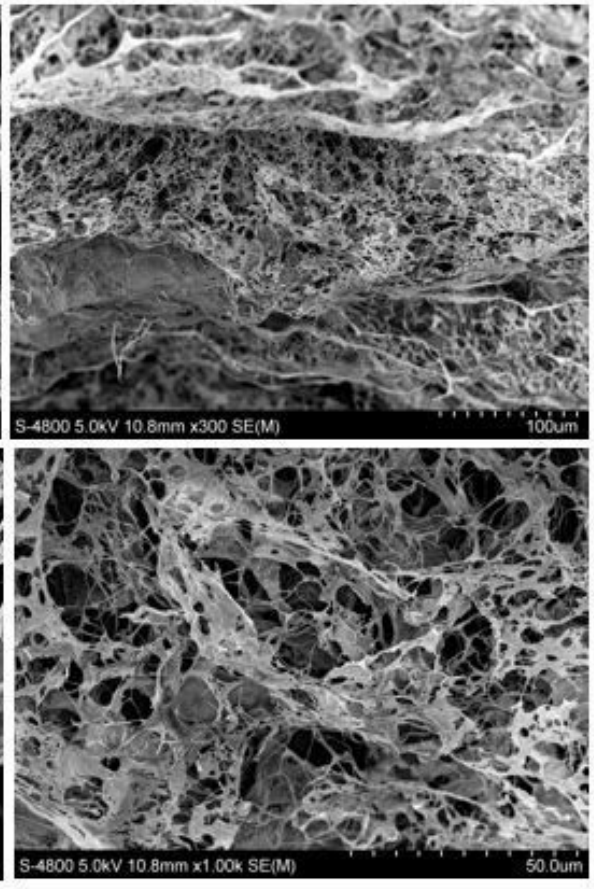

c. $\mathrm{CSH} / \mathrm{n}-\mathrm{HA} / \mathrm{CTS} / \mathrm{VA}$

Figure 6 
Scanning electron microscope (SEM) observation (300x, 1000x). The pore size was 80-200 $\mu \mathrm{m}$, with an average of $140 \mu \mathrm{m}$, and mineral crystallization deposition could be observed in the pore of the VA/CSH/nHA/CTS hydrogel.

\section{Figure 7}

Establishment of rabbit models with chronic osteomyelitis. Gross observation, bacterial culture, Micro-CT and HE staining were carried out indicating typical characteristic of chronic osteomyelitis.

\section{Figure 8}

\section{Morphological evaluation of each group by gross observation at 4 week, 8 weeks and 12 weeks} postoperatively. In control group, typical osteomyelitis and severe tibia damage could be seen (a), and in BMSCs sheet group, defect was partially healed with the infection uncontrolled (b). It could be observed that defects were gradually cured with the absorption of bone substitutes, fibrous tissue was formed, and infection was effectively controlled in Group C and D (c, d).

\section{Figure 9}

3D reconstruction images of the right tibia in each group at 4 week, 8 weeks and 12 weeks after treatment. Reconstruction of defects and infection control were assessed at different time point respectively.

\section{Figure 10}

Quantitative analysis of newborn bone tissue was performed in four groups at 4 week, 8 weeks and 12 weeks after operation (BV/TV, Tb. N, Tb. Th). At 4 weeks, there was a statistically significant difference in new bone formation between the four groups, while up to 12 weeks, there was no difference between the three groups $B, C$, and $D$. 

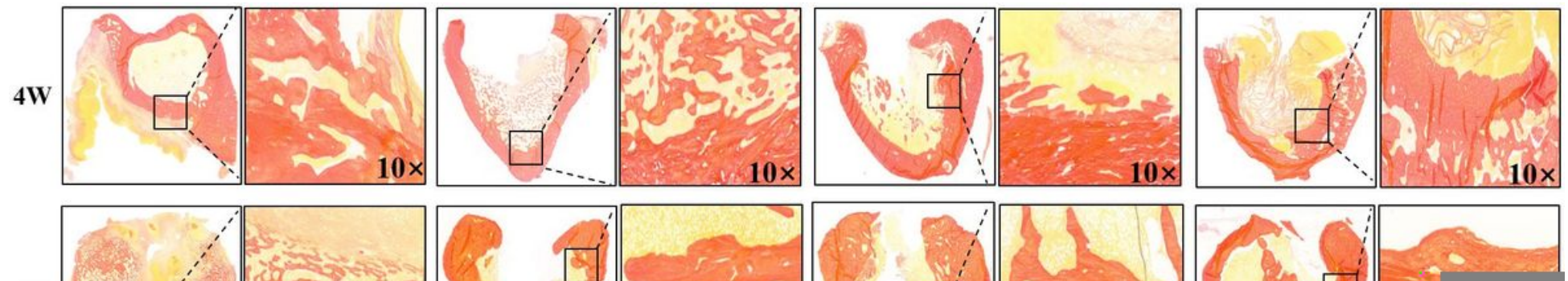

\section{Figure 11}

Histological observation of the four groups at 4, 8, 12 weeks after implantation (Van Gieson staining, 10x).
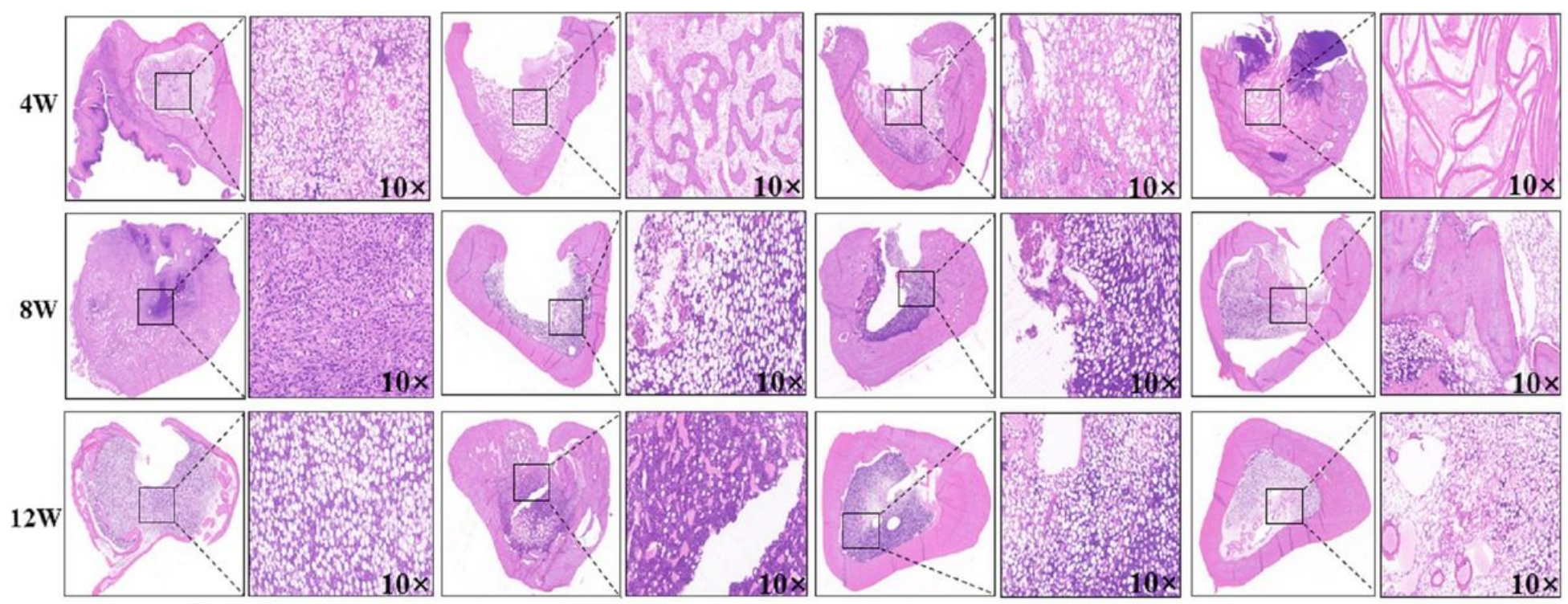

Control

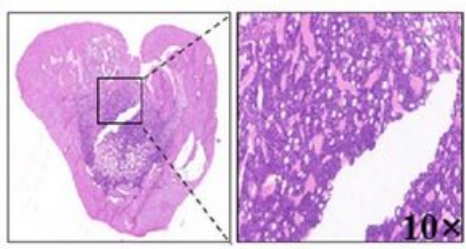

BMSCs Sheet

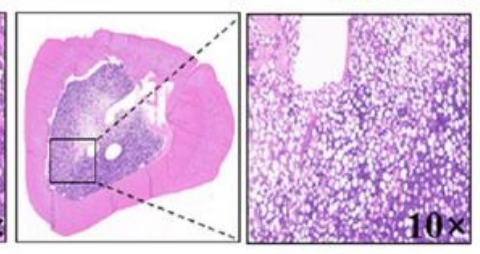

$\mathrm{VA} / \mathrm{CSH} / \mathrm{n}-\mathrm{HA} / \mathrm{CTS}$

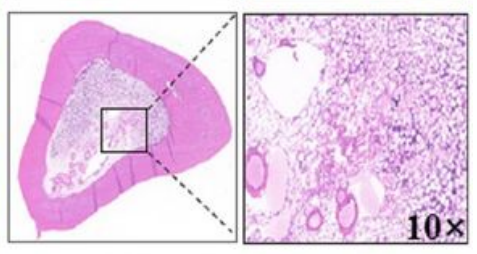

VA/CSH/n-HA/CTS/ BMSCs

Figure 12

Hematoxylin-eosin (HE) staining results of the target site of right tibia of each group at 4, 8, 12 weeks after treatment (10x).

\section{Supplementary Files}

This is a list of supplementary files associated with this preprint. Click to download. 
- CONSORT2010Checklist.doc

Page 23/23 